calibrated to $1.1 \%$ population-level MG prevalence. We modelled the potential impact of using the ResistancePlus MG test (SpeeDx; Sydney, Australia) for symptomatic patients. We conducted sensitivity analyses varying the clearance rate (23-44 days) and percent of infections that are symptomatic in women (0\%-11.7\%).

Results The model estimated a per-partner MG transmission probability of 0.042 . The model predicted that implementing the ResistancePlus test for symptomatic patients would increase the percent of MG infections that are macrolide-susceptible from $50 \%$ to $85 \%$ in 2 years. In sensitivity analyses, transmission probability estimates and 2 year percent of MG infections that are macrolide-susceptible varied from $0.032-0.047$ and 82\%-92\%, respectively.

Conclusion Directing treatment based on aetiology and known macrolide resistance may preserve the ability to treat MG with azithromycin. Moxifloxacin therapy could be limited to patients with known macrolide-resistant MG infection and prevent treatment failure for those patients.

\section{P3.131 AN EMPIRIC RISK SCORE TO GUIDE PRESUMPTIVE TREATMENT OF ASYMPTOMATIC ANORECTAL INFECTIONS IN MEN WHO HAVE SEX WITH MEN IN KISUMU, KENYA}

${ }^{1}$ Laura Quilter, ${ }^{2}$ Eve Obondi, ${ }^{3}$ Colin Kunzweiler, ${ }^{2}$ Duncan Okall, ${ }^{3}$ Robert Bailey, ${ }^{2}$ Fredrick Otieno, 'S Susan Graham. ${ }^{1}$ University of Washington, Seattle, USA; ${ }^{2}$ Nyanza Reproductive Health Society, Kisumu, Kenya; ${ }^{3}$ University of Illinois at Chicago, Chicago, USA

\subsection{6/sextrans-2017-053264.366}

Introduction The World Health Organisation (WHO) recommends presumptive therapy (PT) for Neisseria gonorrhoeae (GC) and Chlamydia trachomatis (CT) in asymptomatic MSM who report unprotected receptive anal intercourse in the past 6 months and either multiple sex partners or a partner with an STI. We aimed to identify predictors of asymptomatic anorectal infection in Kenyan MSM and compare performance of an empiric, model-based risk score to that of the WHO PT algorithm.

Methods Anorectal GC/CT infections were diagnosed at baseline among 698 MSM enrolled in the Anza Mapema study in Kisumu, Kenya. Multivariable logistic regression was used to identify associations with asymptomatic GC/CT anorectal infection. We derived a total risk score (range: 0-5) for each participant using the coefficients of the final multivariable model. Risk score algorithm performance was compared to WHO algorithm performance with respect to sensitivity, specificity, and number needed to treat (NNT).

Results Asymptomatic GC/CT anorectal infection prevalence was $4.2 \%$. Predictors and corresponding risk scores were: HIV infection (2), age 18-24 years (2), and unprotected anal sex (1). A risk score $\geq 3$ was $83 \%$ sensitive and $65 \%$ specific in detecting asymptomatic GC/CT anorectal infection. In contrast, the WHO PT algorithm had low sensitivity (25\%), but was $84 \%$ specific. While $37 \%$ of asymptomatic participants met PT eligibility criteria using a risk score $\geq 3$, only $17 \%$ met eligibility by WHO PT criteria. Using our risk score algorithm, 12 participants would need PT to treat one GC/CT anorectal infection, compared to 38 participants by WHO criteria.

Conclusion An empiric risk score based on age, HIV status, and unprotected anal sex improved both sensitivity and efficiency (i.e., NNT) of identification of asymptomatic GC/CT anorectal infection, compared to the WHO PT algorithm. If validated in other settings, this risk score could improve the management of asymptomatic GC/CT anorectal infections in settings where diagnostic testing is not available.

\section{P3.132 PROJECTING THE EPIDEMIOLOGICAL EFFECT, COST- EFFECTIVENESS AND TRANSMISSION OF HIV DRUG RESISTANCE IN VIETNAM ASSOCIATED WITH VIRAL LOAD MONITORING STRATEGIES}

Lei Zhang. Melbourne Sexual Health Centre, Melbourne, Australia

\subsection{6/sextrans-2017-053264.367}

Introduction Objectives: The objective of this study was to investigate the potential epidemiological impact of viral load (VL) monitoring and its cost-effectiveness in Vietnam, where transmitted HIV drug resistance (TDR) prevalence has increased from $5 \%$ to $5 \%-15 \%$ in the past decade.

Methods Using a population-based mathematical model driven by data from Vietnam, we simulated scenarios of various combinations of VL testing coverage, VL thresholds for secondline ART initiation and availability of HIV drug-resistance tests. We assessed the cost per disability-adjusted life year (DALY) averted for each scenario.

Results Projecting expected ART scale-up levels, to approximately double the number of people on ART by 2030, will lead to an estimated 18510 cases (95\% CI: 9120-34 600 cases) of TDR and 55180 cases (95\% CI: 40540-65 900 cases) of acquired drug resistance (ADR) in the absence of VL monitoring. This projection corresponds to a TDR prevalence of $16 \%$ (95\% CI: $11 \%-24 \%)$ and ADR of $18 \%$ (95\% CI: 15\%-20\%). Annual or biennial VL monitoring with $30 \%$ coverage is expected to relieve $12 \%-31 \%$ of TDR (2260-5860 cases), 25\%-59\% of ADR (9620-22 650 cases), 2\%-6\% of HIV-related deaths (360-880 cases) and 19270-51400 DALYs during 2015-30. The 30\% coverage of VL monitoring is estimated to cost US\$4848-5154 per DALY averted. The projected additional cost for implementing this strategy is US \$105-268 million over 2015-30.

Conclusion Our study suggests that a programmatically achievable $30 \%$ coverage of VL monitoring can have considerable benefits for individuals and leads to population health benefits by reducing the overall national burden of HIV drug resistance. It is marginally cost-effective according to common willingness-to-pay thresholds.

\section{P3.133 EVALUATION OF PRODUCTION AND LYTIC CAPACITY OF VAGINOLYSIN PRODUCED BY BIOTYPES OF GARDNERELLA VAGINALIS}

Lesli Keila Dimas Lopez, Alma Delia Nicolas Morales, Ana Karen Estrada Moreno, Karen Cortés Sarabia, Arturo Ramírez Peralta, Carlos Ortuño Pineda, Amalia Vences Velázquez. Autonomous University of Guerrero, Chilpancingo, Mexico

\subsection{6/sextrans-2017-053264.368}

Introduction: Gardnerella vaginalis is the main etiological agent of bacterial vaginosis (BV), this bacteria has been classified in eight biotypes based on the production of beta-galactosidase, hydrolysis of hippurate and lipase, additionally produces several virulence factors, among them vaginolysin (VLY) is the most important. VLY is a pore-forming cytolysin that damages cells structurally and functionally through the 